\title{
Calculation of neutron background for underground experiments
}

\author{
V. Tomasello ${ }^{1,2}$, V. A. Kudryavtsev ${ }^{1}$, M. Robinson ${ }^{1}$
}

October 22, 2018

${ }^{1}$ Department of Physics and Astronomy, University of Sheffield, Hounsfield Road, Sheffield S3 YRH, $U K$

${ }^{2}$ Physikalisches Institut, Eberhard Karls Universität Tübingen, Auf der Morgenstelle 14, Tübingen D-72076, Germany

Corresponding author:

Vito Tomasello

Department of Physics and Astronomy, University of Sheffield, Hounsfield Road, Sheffield S3 7RH, UK

Tel.: +4411422 23553; fax: +441142223555.

E-mail: v.tomasello@sheffield.ac.uk

\begin{abstract}
New generation dark matter experiments aim at exploring the $10^{-9}-10^{-10}$ $\mathrm{pb}$ cross-section region for the WIMP-nucleon scalar interactions. Neutrons produced in the detector components are one of the main factors that can limit detector sensitivity. Estimation of the background from this source then becomes a crucial task for designing future large-scale detectors. Energy spectra and production rates for neutrons coming from radioactive contamination are required for all materials in and around the detector. In order to estimate neutron yields and spectra, the cross-sections of $(\alpha, n)$ reactions and probabilities of transitions to different excited states should be known. Cross-sections and transition probabilities have been calculated using EMPIRE2.19 for several isotopes, and for some isotopes, a comparison with the experimental data is shown. The results have been used to calculate the neutron spectra from materials using the code SOURCES4A. Neutron background event rates from some detector components in a hypothetical dark matter detector based on Ge crystals have been estimated. Some requirements for the radiopurity of the materials have been deduced from the results of these simulations.
\end{abstract}

PACS: 95.35+d; 14.20.Dh; 13.75.-n; 28.20; 25.40; 98.70.Vc

Keywords: Neutrons; Neutron background; Radioactivity; Dark matter; WIMPs; Underground experiments 


\section{Introduction}

There are many experiments, existing or planned, located deep underground. Such experiments are designed to detect particles coming from astrophysical sources like the WIMPs - Weakly Interacting Massive Particles, or astrophysical neutrinos, or to search for weak processes, for instance neutrinoless double beta decay, predicted by new theories beyond the Standard Model. Underground physics deals with these extremely rare phenomena, which are very difficult to distinguish from other more ordinary signals that come from cosmic rays and natural radioactivity. The best way to screen from cosmic rays is to carry out experiments deep underground, because in this way the surrounding layers of rock reduce their flux by several orders of magnitude $\left(10^{5}-10^{7}\right.$ with respect to the surface). Nevertheless, many radiation backgrounds are still present, primarily originating from radioactivity. The radiation background is crucial for the sensitivity of the aforementioned experiments to expected events. Knowledge of background is therefore essential for designing detectors (in particular for dark matter searches, such as [1, 2, 3, 4, 5, 6, 17, 8]), their shielding and active veto systems, for finding ways of suppressing/rejecting this background and for calculating detector sensitivity. Nowdays in dark matter searches large-scale detectors are required to increase sensitivity to WIMP interactions, since the rate of events is predicted to be very low (between 1 and $10^{-5}$ event $/ \mathrm{kg} /$ day).

One of the main tools for background studies is the use of extensive simulation work. This can help with studying background suppression or rejection strategies, and investigating requirements on the depth, the amount of active/passive shielding, the purity of materials, the veto efficiency, etc. for a given experiment.

There are two main sources of background: local radioactivity and cosmic-ray muons. In this work we will focus only on the radioactivity component.

Radioactive decays result in the production of four types of particles: alphas, electrons/positrons, gamma-rays (X-rays) and neutrons. Alphas and electrons cannot travel far from the source: they quickly lose energy due to ionization and stop within a few microns (alphas) or millimetres (electrons/positrons) from the source. They can generate visible effects in a dark matter detector only if they are produced either within the fiducial volume of the detector or very close to it. Some of the background particles due to natural radioactivity (mainly gamma-rays) can be suppressed by means of passive shielding or rejected by specific identification tools, depending on the experimental design (e.g. electron recoil discrimination for dark matter experiments and single-site selection for neutrinoless $\beta \beta$ decay experiments). Neutrons are more difficult to suppress. Indeed, since single neutron interactions can mimic nuclear recoils produced by WIMPs, their background is crucial in direct dark matter searches.

Neutrons are produced by local radioactivity via spontaneous fission and $(\alpha, n)$ reactions. The $(\alpha, n)$ reactions are initiated by alpha-particles from radioactive decays of uranium and thorium present in minute quantities in rock and detector components. Uranium and thorium decay chains are usually assumed to be in secular equilibrium.

A significant contribution to the neutron flux from spontaneous fission is present only for ${ }^{238} \mathrm{U}$.

To protect a detector from background radiation from rock and laboratory walls, shield- 
ing made of high-Z materials (against gamma-rays) and low-A materials (against neutrons) can be installed around the detector [9, 10, 11, 12, 13, 14, 15. However, the shielding itself and detector components also contain the traces of radioisotopes, becoming sources of background themselves. Materials with radioactive content as low as possible, such as copper for instance, should be used in detector construction. There are also mechanical constraints in choosing the material for the support structure of the detector. Hence a reasonable compromise between mechanical and radioactivity requirements should be found and the calculation of the background rate from detector components is a key factor for any experiment.

Radioactive background transport through the experimental setup is usually carried out using multipurpose particle transport codes, such as GEAnt4[16], MCNPX [17] and FLUKA [18. All these codes are platforms for the simulation of the passage of particles through matter, using Monte Carlo methods. The main inputs for simulations are the energy spectra and production rates of particles of interest. These quantities are not easily obtainable, especially in the case of neutrons. Accurate calculation of neutron yields and spectra, for all materials used in dark matter searches, is crucial for designing experiments and predicting their sensitivities.

The aim of this paper is to present and describe the procedure to calculate neutron yields and spectra from radioactivity for most of materials relevant for dark matter searches. Results from GEANT4 simulations of neutron background will also be shown for the first time for a hypothetical large-scale cryogenic detector based on Ge crystals. The simulations are the starting point for extensive work to be carried out for planning and designing the forthcoming dark matter experiments, such as EURECA (European Underground Rare Event Calorimeter Array) [1]. EuRECA is proposed as a multitarget experiment (scintillators and Ge crystals) but the current work considers only Ge as a target. The aim of future large-scale experiments is to explore scalar WIMP-proton cross-sections in the $10^{-9}-10^{-10} \mathrm{pb}$ region with a target mass of up to one tonne.

We start in Section 2 by calculating $(\alpha, n)$ cross-sections using the code EMPIRE2.19 [19]. Calculation of neutron yields and energy spectra from spontaneous fission and $(\alpha, n)$ reactions using the code Sources4A [20] is discussed in Section 3. These spectra are the essential input to GEANT4 and other particle transport codes for the neutron propagation and detection. We show simulation results for neutrons from radioactivity and their effect on the performance of a hypothetical Ge dark matter detector similar to EURECA.

\section{$2(\alpha, n)$ cross-sections}

A typical background simulation for a dark matter experiment deals with the production and propagation of neutrons and gamma-rays through the experimental setup. The rate of production along with the energy spectra of these particles are required. Spectra of gamma-rays are available from databases, but this is not the case for neutrons. Although neutron yields can easily be estimated (either experimentally or by calculation), if the $(\alpha, n)$ cross section is known as function of energy, the energy spectra of neutrons are difficult to measure or calculate. The measurements of neutron spectra 
are not straightforward since neutrons are neutral particles. Unlike gamma-rays, the neutron spectra depend on the material, so they have to be obtained for all materials relevant to dark matter searches.

The calculation of the neutron spectra requires as input the cross-sections of $(\alpha, n)$ reactions and the transition probabilities to different excited states (branching ratios). As for many isotopes there are no available experimental data, the calculation of these cross-sections is necessary. Even when the data are available, they do not usually include probabilities of transitions to excited states (branching ratios) and therefore cannot be used to generate neutron spectra. For the calculation of $(\alpha, n)$ cross-sections and branching ratios we used the code EMPIRE2.19. We extended the calculations carried out in Ref. [1], by adding more materials and making other improvements.

EMPIRE2.19 is composed of several modules of nuclear reaction codes, including various nuclear models. It is designed for calculations of cross-sections and other parameters over a broad range of energies and incident particles, using any nucleon or heavy ion as the projectile. The code accounts for the major nuclear reaction mechanisms (see Ref. [19] for details). A comprehensive library of input parameters cover nuclear masses, optical model parameters, ground state deformations, discrete level decay schemes, level densities, fission barriers, moments of inertia, and $\gamma$-ray strength functions.

Input to EMPIRE2.19 consists of three parts. The first is mandatory and contains basic data necessary to specify the physics case (incident energy, target, projectile, output particle etc.). This is followed by optional inputs which allow modifications to the default model parameters. In our simulations we used the optical model with Gilber-Cameron level densities adjusted to experimental parameters and to discrete levels. Finally there is the list of incident energies for the projectile ( $\alpha$-particle in our case).

We realized that the results are sensitive to the values of incident alpha energies present in the input file of EMPIRE2.19, for which we wanted to calculate the cross-sections and branching ratios. Choosing a smaller step in energy (bigger number of input energies) results in an improvement in the accuracy. We reduced the step in energy until the variation became insignificant. The step in alpha energy that was used in this work, was $0.1 \mathrm{MeV}$. EmpIRE2.19 is also able to calculate cross-sections for the ground and all excited states. The branching ratios were derived from these cross-sections.

Hence, we achieved significant improvements both for cross-section and branching ratio data over previous calculations [9, 11]. The number of energy points for which the cross-sections and branching ratios were calculated was increased considerably. We also managed to calculate cross-sections for all possible excited states (in the previous work of Ref. [11] some of them were missing).

EMPIRE2.19 also accounts for the continuum spectrum of gamma-rays after the nuclear transmutation. We cannot define an energy value for the continuum, but it is a required input for calculation of neutron yields and spectra. Following the suggestion of the ENDF format user's guide [21] for this case, the sum of the energy threshold of the reaction and the threshold for the continuum level was set as an energy value for the continuum. 
The code Sources4A that was used to calculate neutron yields and spectra as described in Section 3, was changed accordingly to store an increased amount of data in the library.

To prove the reliability of EMPIRE2.19 code, we carried out calculations of $(\alpha, n)$ cross-sections for some isotopes for which experimental data are available. Comparison between EMPIRE2.19 calculations and experimental data is shown in Fig. 1 for ${ }^{54} \mathrm{Fe}$ and ${ }^{62} \mathrm{Ni}$. There is a good agreement between data and simulations, especially for energies below $10 \mathrm{MeV}$, important for alphas from uranium and thorium decay chains. This agreement gives confidence in the EMPIRE2.19 results for others isotopes, for which direct comparison is not possible because of the absence of experimental data.

\section{Neutron yields and spectra}

The evaluation of neutron yields and spectra can be carried out using the code SouRCES4A. The code calculates neutron yields and spectra from $(\alpha, n)$ reactions, spontaneous fission, and delayed neutron emission due to the decay of radionuclides. Its library contains all alpha emission lines from most radioactive isotopes. The code takes into account the energy losses of alphas, cross-sections of $(\alpha, n)$ reactions and the probabilities of nuclear transition to different excited states. We used an option of thick target neutron yield that allows calculation of neutron yields and spectra under the assumption that the size of a radioactive sample exceeds significantly the range of alphas.

Despite the tested reliability (see [20, 23]), the way in which it was conceived presented two limitations. The first comes from the fact that the original code provided a treatment of $(\alpha, n)$ reactions only up to $6.5 \mathrm{MeV}$. This restricted significantly the reliability of the results, because the cross-section of $(\alpha, n)$ reactions rises with energy and the average neutron energy also increases with the parent alpha energy. Therefore a modified version of Sources4A [9] was used in this work. In this version, alpha energies up to $10 \mathrm{MeV}$ are allowed. Another problem with original Sources4A was that the cross-sections and branching ratios for transitions to the excited states for many isotopes relevant to dark matter searches were missing from the code libraries.

As described in Section 2, cross-sections and branching ratios for several isotopes were calculated with EMPIRE2.19 and inserted into the libraries of SourCES4A. The code was then used to calculate neutron yields and spectra from decay chains of $U$ and Th for many materials present in rocks and possible detector components. If needed, other materials can easily be added. Usually a concentration of 1 ppb of $U$ or 1 ppb of Th in all calculations was assumed, which can easily be scaled to the actual concentrations. In all calculations so far the $U$ and Th decay chains were assumed to be in secular equilibrium.

The most critical materials to be considered as neutron sources, are mainly: (1) rock and concrete in the walls of the underground laboratories, that dominate the total neutron flux before shielding, and (2) materials that compose the internal detector parts (e.g. stainless steel, copper, photomultiplier tubes etc.), that become important when the external flux is attenuated by the shielding. Following these criteria, neutron 
spectra shown in this section are: copper, stainless steel, the rock around the Modane Underground Laboratory (France) and some typical materials (teflon and polyethylene) present among the components of experimental setups.

The spectra from stainless steel are presented in Fig. 2 a, showing a significant contribution from spontaneous fission from ${ }^{238} \mathrm{U}$. Fig. 2 $\mathrm{b}$ shows comparison of our calculations for stainless steel using $1 \mathrm{ppb}$ of $U$ and Th with a previous work [9]. In Ref. [9] the neutron yields were also calculated with SouRCES4A, but the library contained a restricted set of isotopes and transitions for many isotopes were assumed to the ground state only. For instance, cross-sections for all Fe isotopes were assumed to be the same as the measured cross-section for ${ }^{54} \mathrm{Fe}$. Fig. 2b shows that these assumptions led to the overestimation of the neutron energies and underestimation of the neutron flux.

The neutron energy spectra from $U$ and Th in copper are shown in Fig. 3 . A logarithmic scale on $\mathrm{y}$-axis is chosen here to make visible the small contribution from $(\alpha, n)$ reactions. The neutron yield from $(\alpha, n)$ reactions is suppressed in copper due to the high energy threshold which is about $7 \mathrm{MeV}$ (due to high- $\mathrm{Z}$ and Coulomb barrier). In this case the main contribution comes from spontaneous fission of ${ }^{238} \mathrm{U}$. Hence, for a given $\mathrm{U} / \mathrm{Th}$ concentration, copper has an advantage over other materials containing lower-Z isotopes (for instance stainless steel).

Fig. 4 shows the neutron energy spectrum at production in rock around the Modane Underground Laboratory. It is similar to that presented in Ref. [11, but has been obtained with the aforementioned improvements to the $(\alpha, n)$ cross-section calculations. The rock composition and U/Th concentrations were taken from Ref. [24]. Separate contributions from $(\alpha, n)$ reactions in the uranium decay chain, thorium chain and ${ }^{238} \mathrm{U}$ spontaneous fission are shown. The spectrum of neutrons from ${ }^{238} \mathrm{U}$ spontaneous fission is described by the Watt function [25], with a peak energy of about $0.8 \mathrm{MeV}$ and a mean energy of about $1.7 \mathrm{MeV}$. As the spectra of neutrons from $(\alpha, n)$ reactions are not much harder, this results in an overall energy spectrum with mean energy of about $1.9 \mathrm{MeV}$. In general, simulations with SoURCES4A, taking into account proper branching ratios for transitions to excited states, give softer neutron spectra than reported in earlier simulations and measurements (see, for instance, Refs. [24, 26]).

Fig. 5]and Fig. 6 show neutron energy spectra from U and Th in teflon and polyethylene. In both cases spontaneous fission is not the main contributor. Its contribution is practically negligible for teflon. These two materials are often used in small amounts nearby the detectors. The background neutron flux from materials close to the sensitive volume of the detector is difficult to shield or suppress by active rejection techniques. It is, therefore, crucial to estimate the contribution of these materials to the background event rate since they can limit the sensitivity of the experiments.

\section{Neutron background from detector components}

In this Section we present some results of neutron propagation and detection in a particle dark matter detector based on Ge. We used neutron yields and energy spectra, as calculated using the modified version of SOURCES4A with extended libraries, as input to the simulations. These simulations form a part of a preliminary study to investigate 
radioactive background for future large-scale cryogenic dark matter detectors.

Dark matter detectors are looking for low energy depositions (keV energies) from nuclear recoils due to WIMP-nucleus interactions. Gamma-ray flux from radioactivity usually exceeds by several orders of magnitude the neutron flux. However, gammarays generate electron recoils in dark matter detectors that can relatively easily be discriminated from nuclear recoils expected from WIMPs.

Neutrons are more likely to cause problems than gamma-rays since they can elastically scatter off nuclei producing nuclear recoils in a similar way to WIMPs.

The simulations described here were mainly intended to study:

1. The background event rate due to neutrons from copper - the most massive material in the cryogenic detector construction.

2. The change in background rate due to neutrons if stainless steel is used in addition to copper.

The simulations were carried out using GEANT4. Fig. 7 shows the detector consisting of two copper vessels (inner vessel with thickness of $0.5 \mathrm{~cm}$ and a mass of $139 \mathrm{~kg}$, and an outer vessel with a thickness of $0.5 \mathrm{~cm}$ and a mass of $181 \mathrm{~kg}$ ) containing $103.68 \mathrm{~kg}$ of $\mathrm{Ge}$ supported by a copper plate with a thickness of $1 \mathrm{~cm}$ and a mass of $22 \mathrm{~kg}$. Crystals are arranged in 27 floors, each containing 12 crystals with a mass of $320 \mathrm{~g}$ each. Crystals are similar to those described in Ref. [27. Although we used a simplified design of a dark matter detector based on Ge target, it enabled us to estimate the background rates due to neutrons from the most massive detector components.

Neutrons were generated evenly within two copper vessels and a copper plate below the Ge crystals. Their directions were sampled isotropically. Neutrons were transported to the crystals using GEANT4 toolkit and their energy depositions in Ge were recorded. Both elastic and inelastic scattering of neutrons were taken into account. As a result recorded energy depositions were due to nuclear recoils and/or gamma-rays/electrons produced via neutron inelastic scattering in crystals or their surroundings. In further analysis we considered only events with energy deposition in a single crystal more than $10 \mathrm{keV}$. This energy corresponds approximately to the expected threshold of highsensitivity detectors.

Fig. 8 shows an energy spectrum of single nuclear recoils above $10 \mathrm{keV}$ in Ge from 1 $\mathrm{ppb}$ of uranium and thorium in the two vessels and the copper plate supporting the crystals. To plot this spectrum the following selections have been made: (i) only single nuclear recoils have been selected, i.e. if a neutron scattered more than once producing two or more nuclear recoils in two or more different crystals with energies more than 10 $\mathrm{keV}$ each, the event was rejected; (ii) if two scatters happened in one crystal, they were considered as a single nuclear recoil, i.e. no position sensitivity was assumed within a single crystal; (iii) if an energy deposition in a crystal was less than $10 \mathrm{keV}$, it was neglected, i.e. an energy threshold for a deposition in a crystal was set to $10 \mathrm{keV}$; (iv) if an energy deposition was due to an electron recoil either alone or in combination with a nuclear recoil, it was neglected, i.e. a perfect discrimination was assumed for gamma-ray induced events. The spectrum shown in Fig. 8 was obtained assuming 1 
ppb concentrations of $U$ and Th in copper that is probably 100 times higher than could be achieved for ultra-low radioactivity copper.

Fig. 9 shows the multiplicity distribution of events that include nuclear recoils. Neutrons were produced by the uranium and thorium decay chains ( 1 ppb of $U$ and $T h$ ) in the copper vessels and supporting plate. A single energy deposition (multiplicity equal to one) was defined in the same way as above. If more than one "energy deposition" due to either nuclear or electron recoils occurred in different crystals, then an appropriate multiplicity was attributed to this event. Energy depositions from electron recoils (due to neutron inelastic scattering) were included in the plot. If an energy deposition in a crystal was less than $10 \mathrm{keV}$, it was considered as undetected. More than $60 \%$ of energy depositions associated with neutrons were due to single nuclear recoils. It is expected that events with multiple energy depositions in different crystals will be rejected by the data acquisition system or off-line analysis.

Table 1: Background event rate per year at $10-50 \mathrm{keV}$ due to single nuclear recoils from neutron interactions in $103.68 \mathrm{~kg}$ of Ge. Neutron yield at production in the first column is given in neutrons $/ \mathrm{s} / \mathrm{cm}^{3}$.

\begin{tabular}{|c|c|c|c|}
\hline $\begin{array}{c}\text { Radioactive } \\
\text { contamination }\end{array}$ & $\begin{array}{c}\text { Inner Cu vessel } \\
(\text { mass }=139 \mathrm{~kg})\end{array}$ & $\begin{array}{c}\text { External Cu vessel } \\
(\text { mass }=181 \mathrm{~kg})\end{array}$ & $\begin{array}{c}\text { Plate } \\
(\mathrm{Cu} \text { mass }=22.1 \mathrm{~kg} \text { or } \\
\text { steel mass }=19.7 \mathrm{~kg})\end{array}$ \\
\hline $\begin{array}{c}1 \mathrm{ppb} \mathrm{U} \text { in } \mathrm{Cu} \\
\left(\mathrm{n} \text { yield }=1.24 \times 10^{-10}\right)\end{array}$ & 1.49 & 1.35 & 0.680 \\
\hline $\begin{array}{c}1 \mathrm{ppb} \mathrm{Th} \text { in } \mathrm{Cu} \\
\left(\mathrm{n} \text { yield }=8.39 \times 10^{-12}\right)\end{array}$ & 0.130 & 0.114 & 0.0586 \\
\hline $\begin{array}{c}1 \mathrm{ppb} \mathrm{U} \text { in stainless steel } \\
\left(\mathrm{n} \text { yield }=1.47 \times 10^{-10}\right)\end{array}$ & & & 0.812 \\
\hline $\begin{array}{c}1 \mathrm{ppb} \mathrm{Th} \text { in stainless steel } \\
\left(\mathrm{n} \text { yield }=5.16 \times 10^{-11}\right)\end{array}$ & & & 0.284 \\
\hline
\end{tabular}

To quantify the sensitivity of the experiment to WIMPs and limitations due to various backgrounds, we defined an energy range of $10-50 \mathrm{keV}$ as the range for the data analysis from the future experiment. Table 1 shows the expected event rate due to single nuclear recoils in the 10-50 keV energy range. Four different components were considered: inner copper vessel, outer copper vessel, copper plate, stainless steel plate. In the latter case, the copper plate was substituted with a similar plate made of stainless steel. In all cases $1 \mathrm{ppb} \mathrm{U}$ and Th was assumed. Stainless steel gives a slightly higher rate of events than copper due to higher cross-sections of $(\alpha, n)$ reactions on some isotopes, assuming the same concentrations of $U$ and Th. It is known, however, that the usual concentrations of $U$ and Th in stainless steel are around 1 ppb whereas copper can be made 100 times less contaminated with radioactive isotopes. Table 1 shows that about $20 \mathrm{~kg}$ of stainless steel can produce a non-negligible neutron event rate in the detector. This leads to the conclusion that no more than a few $\mathrm{kg}$ of steel should be allowed to be used in the detector construction. The mass of copper and its contamination should 
also be reduced to a minimum. A few thousand $\mathrm{kg}$ of copper with a concentration of around $0.01 \mathrm{ppb}$ of $\mathrm{U}$ and $\mathrm{Th}$ do not limit the sensitivity of the detector made of 100 $\mathrm{kg}$ of Ge. Values given in Table 1 can be scaled up/down for different masses of copper (stainless steel) and different concentrations of radioactive isotopes. Scaling with the target mass or distance between copper and crystals is not straightforward. To obtain accurate values, simulations should be repeated for different designs.

\section{Conclusions}

This work aimed mainly at presenting a tool for calculating neutron background associated with various radioactive isotope concentrations in different materials. This was done using the code SourCES4A, the libraries of which were extended by adding a large number of cross-sections for $(\alpha, n)$ reactions and branching ratios. Cross-sections for $(\alpha, n)$ reactions and branching ratios were evaluated using the code EMPIRE2.19. Neutron yields and spectra from uranium and thorium decay chains in different materials used in dark matter detectors and present in the rocks surrounding the experimental underground laboratories have been obtained. The radioactive contaminations of $\mathrm{U}$ and Th assumed for the calculations can easily be scaled to the actual values. If needed, spectra from other materials can be calculated using the same method.

We also showed first results concerning the estimated neutron background in a future large-scale dark matter experiment, based on cryogenic Ge detectors such as EuRECA. Based on the simulations carried out in present work, conclusions can be derived that can help with the baseline design of the Ge experiment expected to reach a sensitivity of about $10^{-10} \mathrm{pb}$ to WIMP-nucleon cross-section:

1. A few tonnes of low radioactivity copper $(\leq 0.01 \mathrm{ppb} \mathrm{U} / \mathrm{Th})$ can be used in the detector construction. This will produce a neutron background rate of less than 1 event per year at $10-50 \mathrm{keV}$ and not limit detector sensitivity to WIMPs.

2. No more than a few $\mathrm{kg}$ of stainless steel or other material with concentrations of about $1 \mathrm{ppb}$ of $\mathrm{U} / \mathrm{Th}$ are allowed inside the shielding.

3. Materials with higher concentrations of radioactive isotopes (more than $1 \mathrm{ppb}$ of $\mathrm{U} / \mathrm{Th}$ ) should be avoided or their mass should be limited to less than a few $\mathrm{kg}$.

\section{Acknowledgments}

This work has been supported by the ILIAS integrating activity (Contract No. RII3CT-2004-506222) as part of the EU FP6 programme in Astroparticle Physics. One of us (VT) would like to thank ILIAS for the financial support of his $\mathrm{PhD}$ research. We acknowledge also the support from the Science and Technology Facility Council (UK). We would like to thank the members of the EURECA Collaboration for fruitful discussions. 


\section{References}

[1] H. Kraus et al., Nucl. Phys. Proc. Suppl. 173 (2007) 168.

[2] T. Bruch (CDMS Collaboration), AIP Conf. Proc. 957 (2007) 193.

[3] E. Aprile et al., Nucl. Phys. Proc. Suppl. 173 (2007) 113 (Erratum-ibid. 175-176 (2008) E1).

[4] R. Brunetti et al. (WARP Collaboration), New Astron. Rev. 49 (2005) 265 (arXiv:astro-ph/0405342).

[5] L. Kaufmann and A. Rubbia, Nucl. Phys. Proc. Suppl. 173 (2007) 141.

[6] D. Muna (for the DRIFT Collaboration), Nucl. Phys. Proc. Suppl. 173 (2007) 172.

[7] M. Tripathi (for the LUX Collaboration), talk at the conference: General INPAC meeting May 4-6 2007, Berkeley, CA, USA (http://cosmology.berkeley.edu/inpac/INPAC_May07/Talks/Tripathi_LUX_INPAC.pdf).

[8] N. J. T. Smith, talk at the conference: 13th International Symposium On Particles, Strings And Cosmology (PASCOS 07) 2-7 Jul 2007, London, England (http://www.pascos07.org/programme/talks/Smith.pdf).

[9] M. J. Carson et al., Astropart. Phys. 21 (2004) 667.

[10] H. M. Araújo, V. A. Kudryavtsev, N. J. C. Spooner and T. J. Sumner., Nucl. Instrum. \& Meth. in Phys. Res. A 545 (2005) 398.

[11] R. Lemrani et al., Nucl. Instr. and Meth. in Phys. Res. A 560 (2006) 454.

[12] D. Y. Stewart, P. F. Harrison, B. Morgan and Y. Ramachers, Nucl. Instr. and Meth. A 571 (2007) 651.

[13] D. S. Akerib et al., arXiv:astro-ph/0605719.

[14] H. Wulandari, J. Jochum, W. Rau and F. von Feilitzsch, arXiv:hep-ex/0401032.

[15] S. Fiorucci et al. (EDELWEISS Collaboration), Astropart. Phys. 28 (2007) 143.

[16] S. Agostinelli et al. (GEANT4 Collaboration), Nucl. Instr. and Meth.A 06 (2003) 250.

[17] Mcnpx, website http://mcnpx.lanl.gov.

[18] A. Fassò et al., FLUKA: a multi-particle transport code, CERN-2005-10 (2005), INFN/TC-05/11, SLAC-R-773; A. Fassò et al., Computing in High Energy and Nuclear Physics 2003 Conference (CHEP2003), La Jolla, CA, USA, March 24-28, 2003, (paper MOMT005) eConf C0303241, hep-ph/0306267. 
[19] M. Herman, R. Capote, B. Carlson, P. Obložinský, M. Sin, A. Trkov, H. Wienke, V. Zerkin, EMPIRE: Nuclear Reaction Model Code System for Data Evaluation Nucl. Data Sheets 108 (2007) 2655; EmpIRE 2.19 UsER's Guide, www.nndc.bnl.gov/empire219/downloads.html.

[20] W.B. Wilson et. al., SOURCES4A: A Code for Calculating $(\alpha, n)$, Spontaneous Fission, and Delayed Neutron Sources and Spectra, Technical Report LA-13639MS, Los Alamos (1999).

[21] ENDF-6 Formats User's Guide, www-nds.iaea.org/ndspub/documents/endf/endf102/.

[22] ExFOR, http://www.nndc.bnl.gov/exfor/exfor00.htm.

[23] V.A.Kudryavtsev, Proc. of the Topical Workshop on Low Radioactivity Techniques (Sudbury, Canada), AIP Conference Proceedings vol 785, ed B Cleveland, R Ford and M Chen (New York: Melville), 2005, p 238.

[24] V. Chazal et al., Astropart. Phys. 9 (1998) 163.

[25] B.E. Watt, Phys. Rev. 87 (1952) 1037.

[26] F. Arneodo et al., Nuovo Cimento A 112 (1999) 819.

[27] X. F. Navick, M. Chapellier, F. Deliot, S. Herve and L. Miramonti, Nucl. Instr. and Meth. A 444 (2000) 361. 


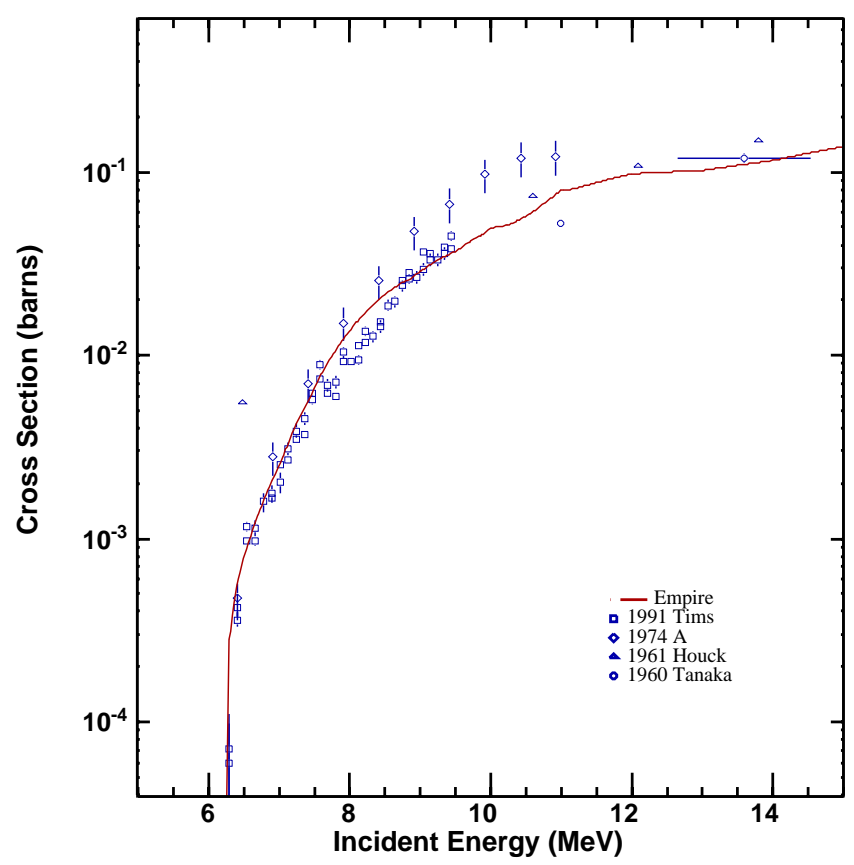

(a)

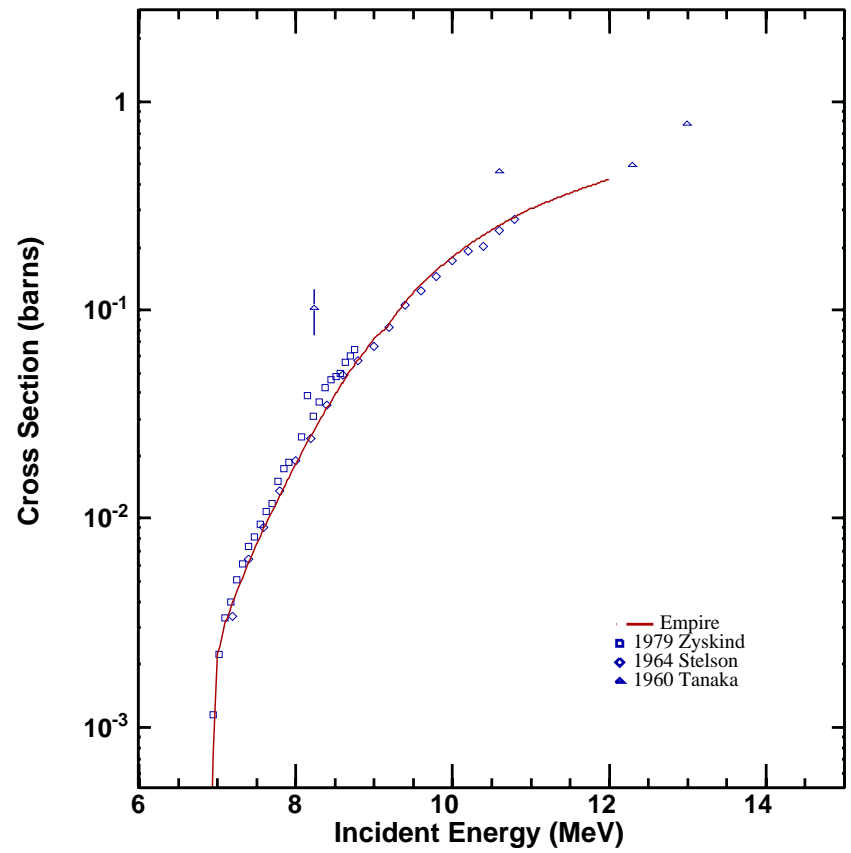

(b)

Figure 1: $(\alpha, n)$ cross-sections as a function of alpha energy. Results obtained with EMPIRE2.19 (solid curve) are compared with experimental data: (a) ${ }^{54} \mathrm{Fe}$ and (b) ${ }^{62} \mathrm{Ni}$. For the experimental data the ExFor [22] library was used. 


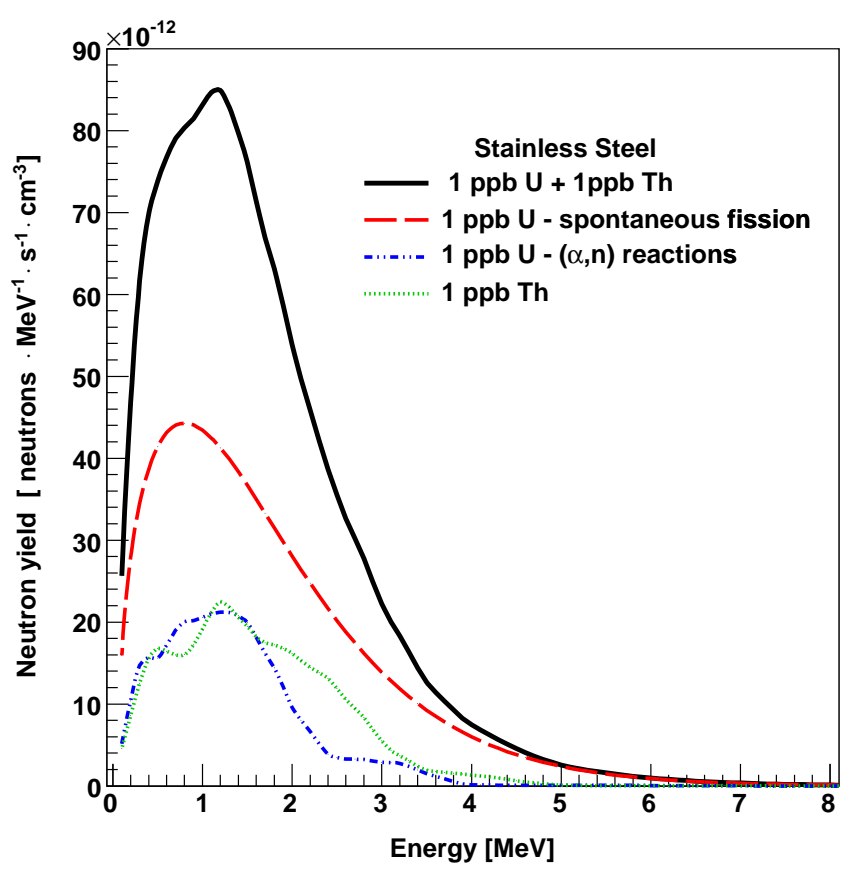

(a)

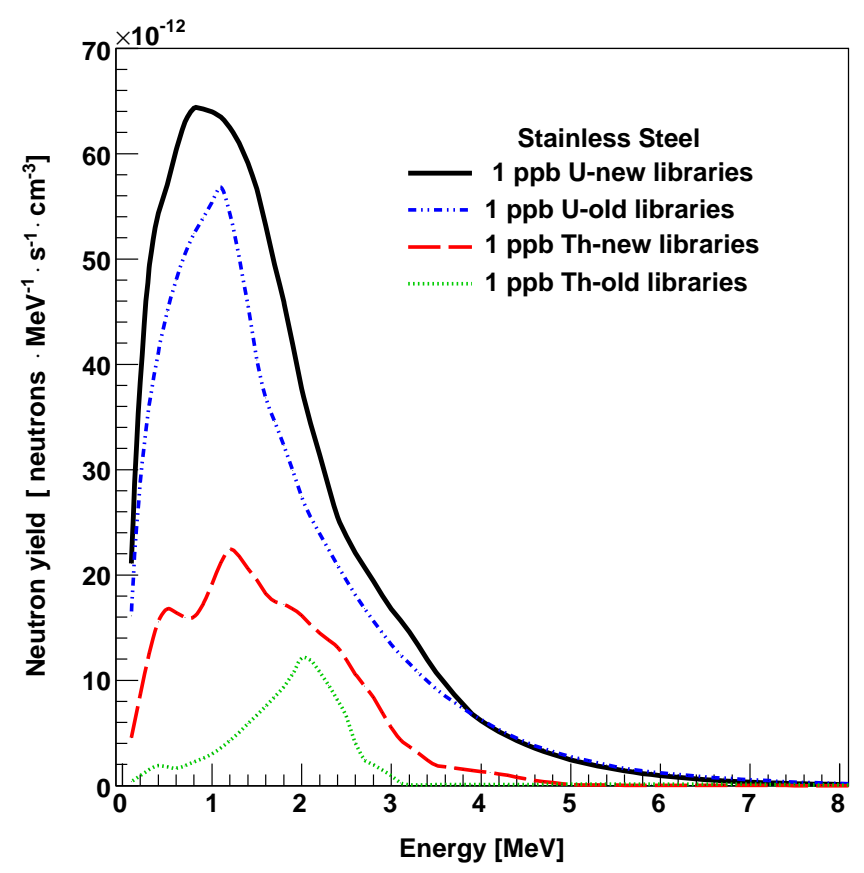

(b)

Figure 2: (a) Neutron spectra from stainless steel with 1 ppb of $U$ and 1 ppb of Th. (b) The spectra from present work are compared to those calculated using different Sources4A libraries. The "new" library refers to our present results, the "old" library refers to Ref. [9]. The neutron yields for $1 \mathrm{ppb}$ of $U$ and $1 \mathrm{ppb}$ of Th are $1.47 \times 10^{-9}$ neutrons $\cdot \mathrm{s}^{-1} \cdot \mathrm{cm}^{-3}(\mathrm{U})$ and $4.74 \times 10^{-10}$ neutrons $\cdot \mathrm{s}^{-1} \cdot \mathrm{cm}^{-3}$ (Th) for "new" library, and $1.19 \times 10^{-9}$ neutrons $\cdot \mathrm{s}^{-1} \cdot \mathrm{cm}^{-3}(\mathrm{U})$ and $1.56 \times 10^{-10}$ neutrons $\cdot \mathrm{s}^{-1} \cdot \mathrm{cm}^{-3}(\mathrm{Th})$ for the old ones. The mean neutron energies are: $<E_{n}>=1.58 \mathrm{MeV}(\mathrm{U}),<E_{n}>=1.56 \mathrm{MeV}$ (Th) for the new libraries and $<E_{n}>=1.66 \mathrm{MeV}(\mathrm{U}),<E_{n}>=1.92 \mathrm{MeV}$ (Th) for the old ones. See text for details. 


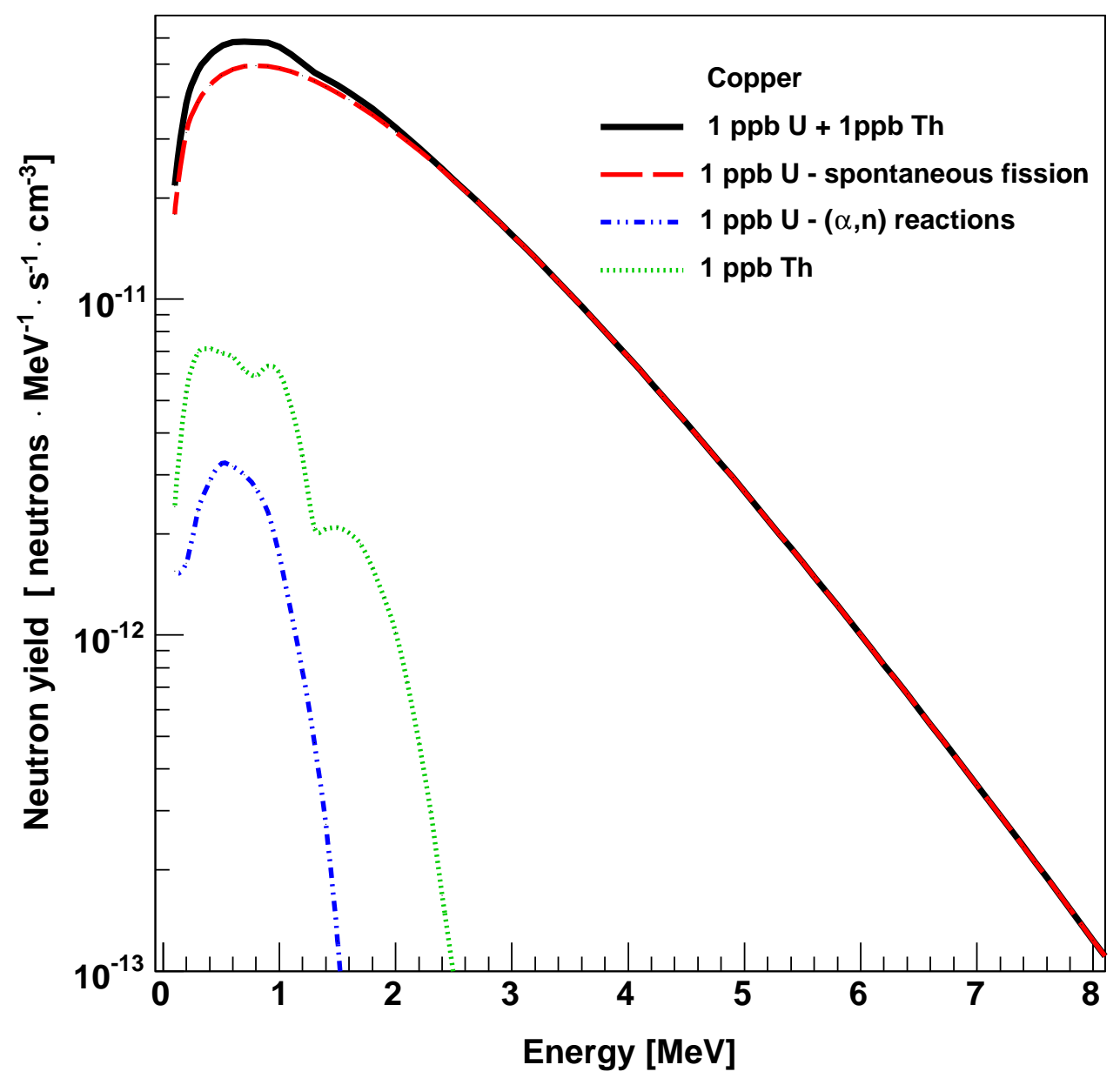

Figure 3: Neutron spectra from copper assuming $1 \mathrm{ppb}$ (both $\mathrm{U}$ and Th) of radioactive contamination. Different contributions are shown along with the sum spectrum (solid curve). 


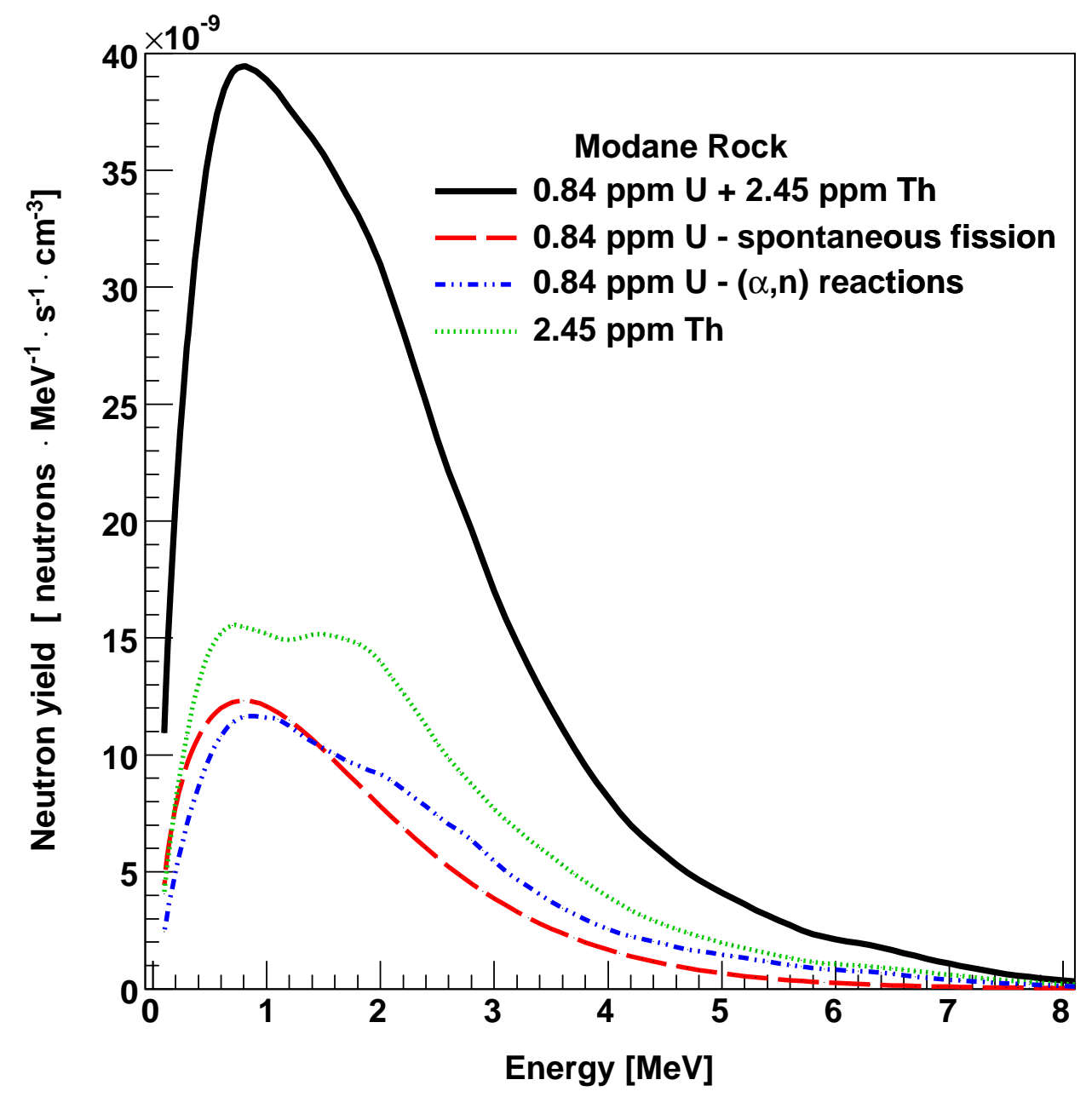

Figure 4: Neutron energy spectra from uranium and thorium in the rock around the Modane Underground Laboratory. Rock contamination is taken from [24]. Different contributions are shown along with the sum spectrum (solid curve). 


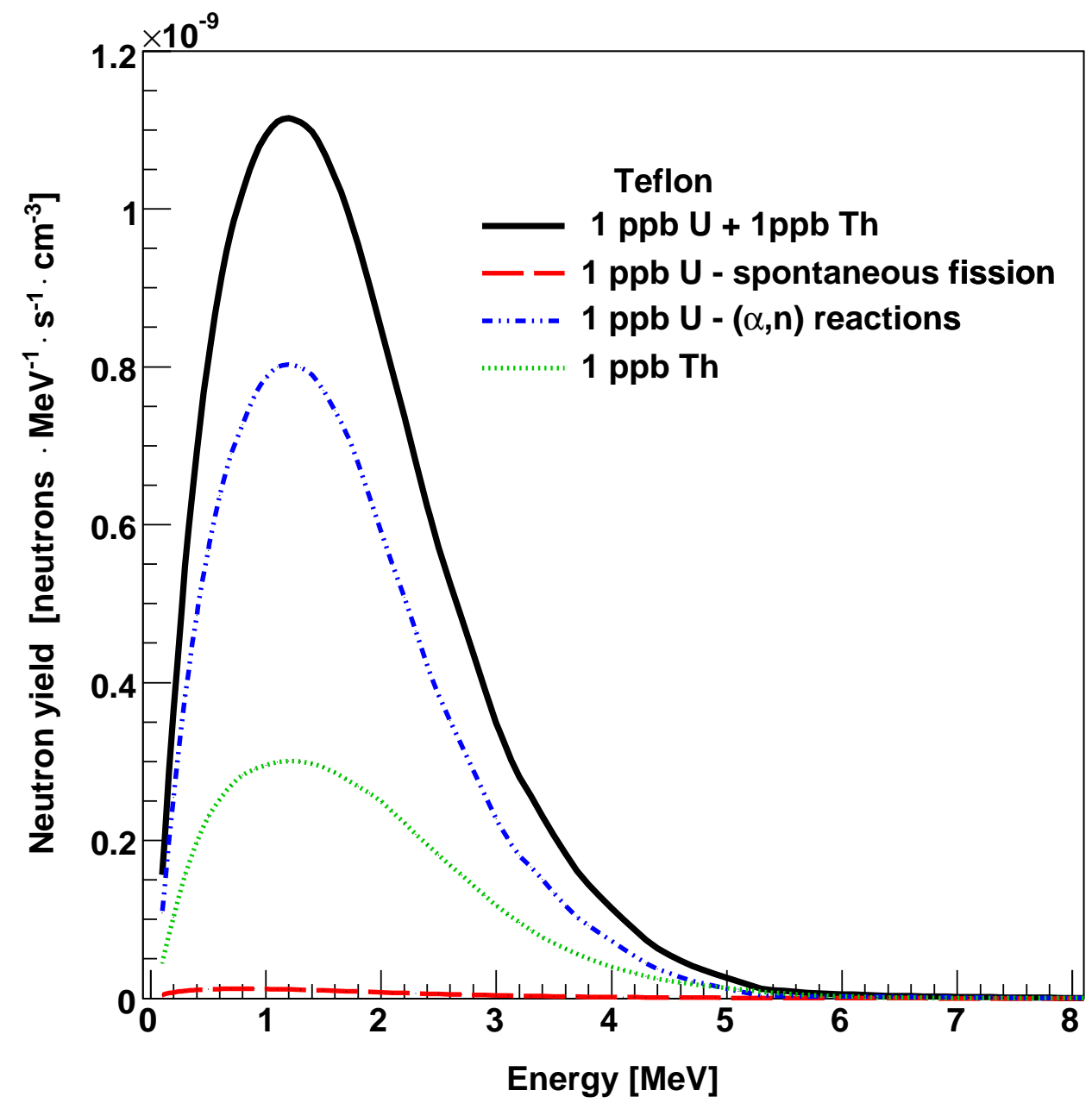

Figure 5: Neutron spectra from teflon assuming $1 \mathrm{ppb}$ (both $\mathrm{U}$ and $\mathrm{Th}$ ) of radioactive contamination. Different contributions are shown along with the sum spectrum (solid curve). 


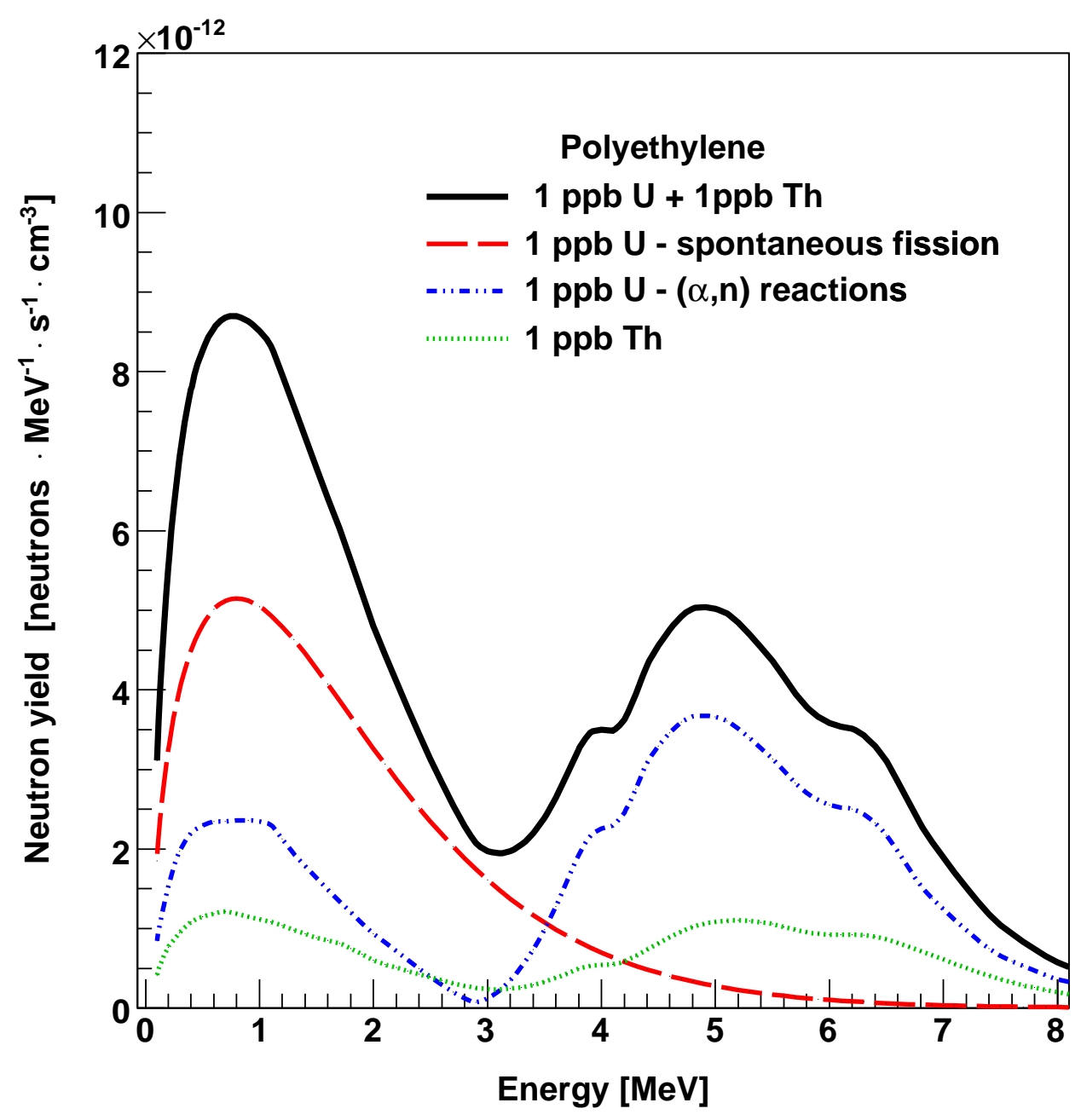

Figure 6: Neutron spectra from polyethylene assuming $1 \mathrm{ppb}$ (both U and Th) of radioactive contamination. Different contributions are shown along with the sum spectrum (solid curve). 


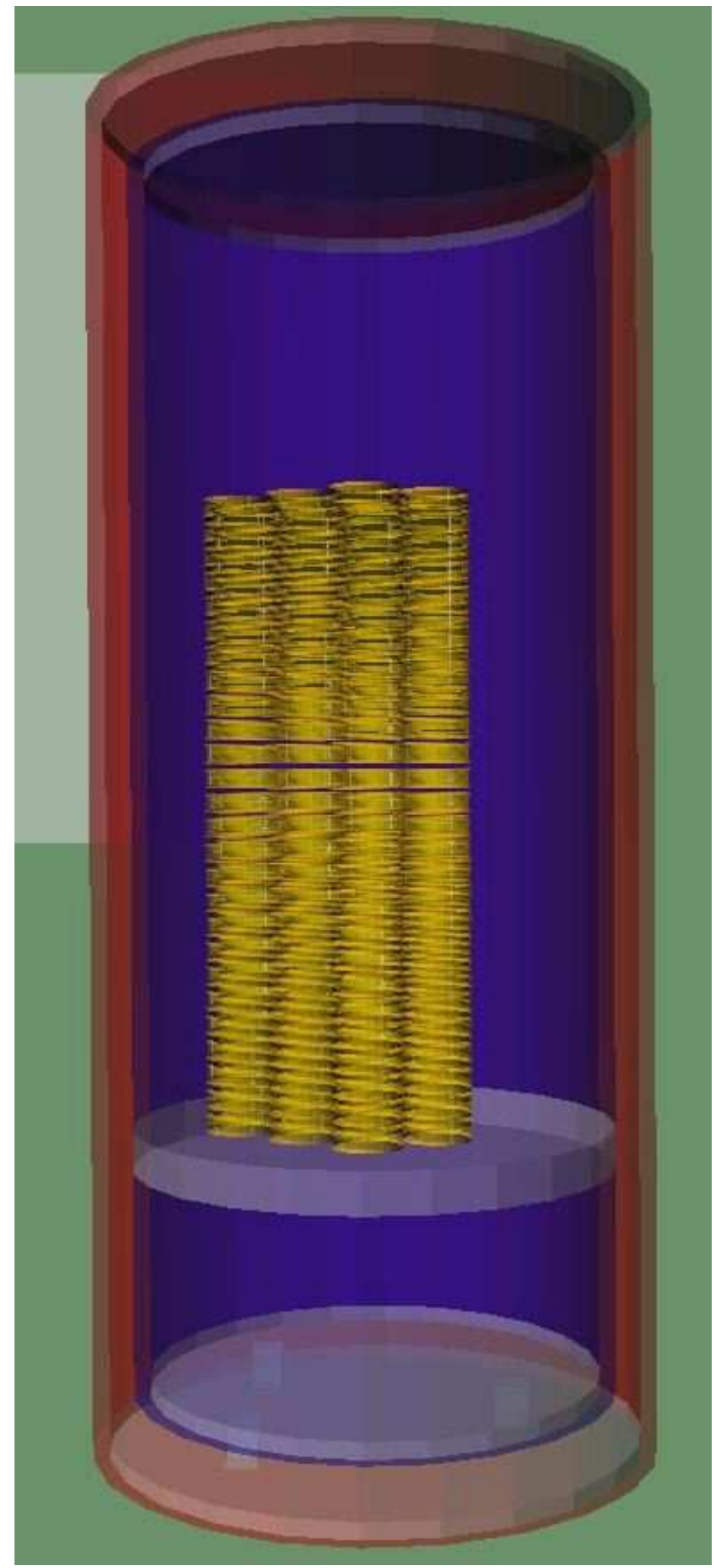

Figure 7: Schematic view of copper vessels and Ge crystals from GEANT4. 


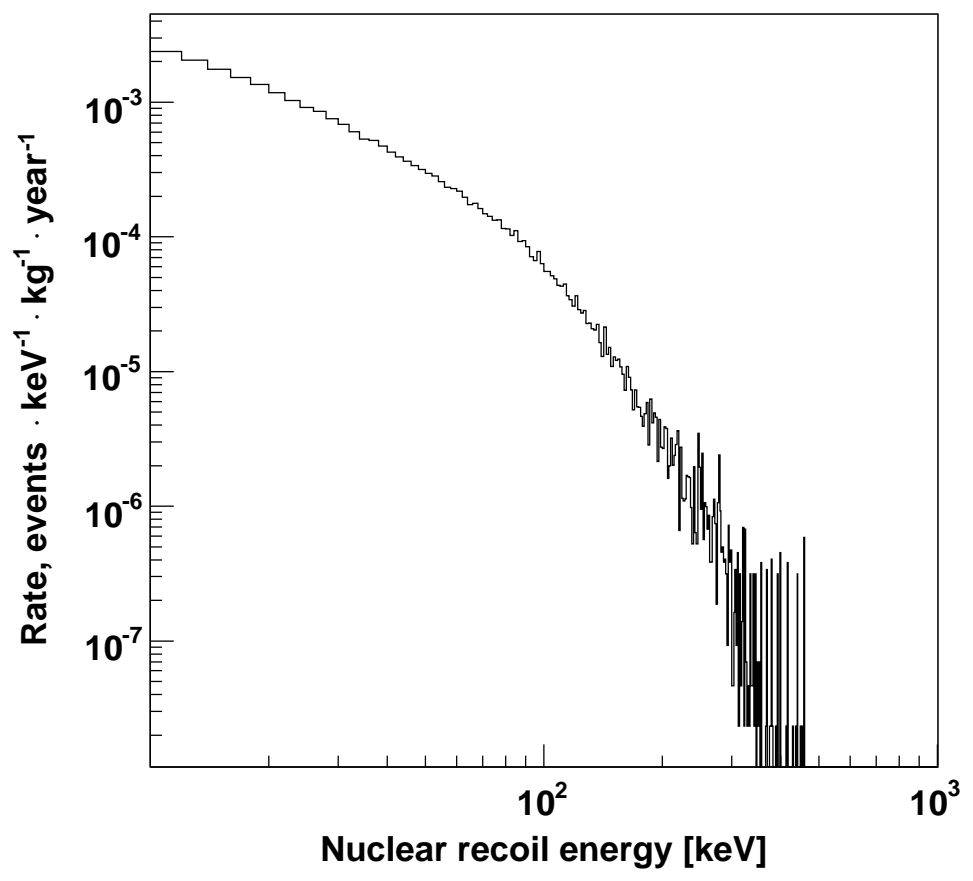

Figure 8: Energy spectrum of nuclear recoils in $103.68 \mathrm{~kg}$ of Ge from $1 \mathrm{ppb}$ of uranium and thorium in the copper vessels and plate. The energy threshold of $10 \mathrm{keV}$ was assumed to select events (see text for details). 


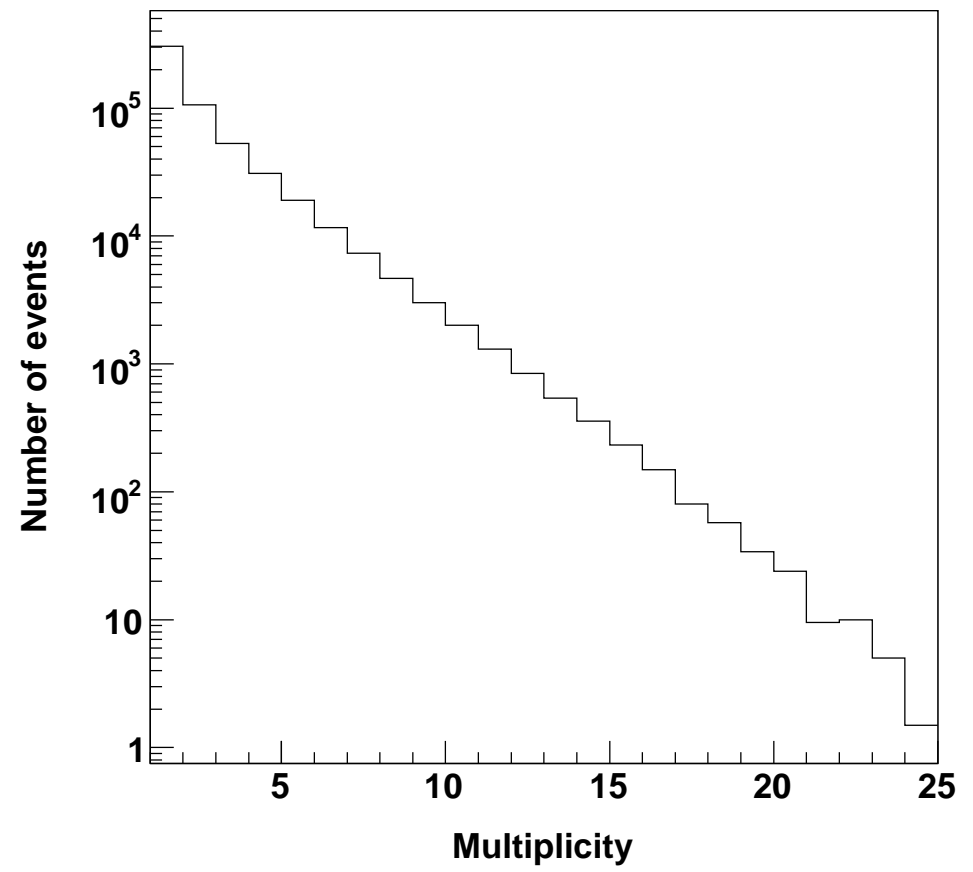

Figure 9: Multiplicity distribution of energy depositions due to nuclear recoils in Ge crystals from $1 \mathrm{ppb}$ of uranium and thorium in the copper vessels and plate. Energy depositions from nuclear and electron recoils are included but at least one nuclear recoil above $10 \mathrm{keV}$ is required. The energy threshold of $10 \mathrm{keV}$ was assumed to select events (see text for details). 